\title{
Cellular nucleic acid-binding protein is vital to testis development and spermatogenesis in mice
}

\author{
Bo Zheng ${ }^{1,2, *}$, Jun $\mathrm{Yu}^{2,3, *}$, Yueshuai Guo ${ }^{2,4, *}$, Tingting $\mathrm{Gao}^{2,5}$, Cong Shen ${ }^{1,2}$, Xi Zhang ${ }^{2}$, \\ Hong $\mathrm{Li}^{1}$ and Xiaoyan Huang ${ }^{2}$ \\ ${ }^{1}$ Center for Reproduction and Genetics, Suzhou Municipal Hospital, The Affiliated Suzhou Hospital of Nanjing \\ Medical University, Suzhou, China, ${ }^{2}$ State Key Laboratory of Reproductive Medicine, Department of Histology and \\ Embryology, Nanjing Medical University, Nanjing, China, ${ }^{3}$ Department of Obstetrics and Gynecology, Affiliated \\ Hospital of Jiangsu University, Jiangsu University, Zhenjiang, China, ${ }^{4}$ The Affiliated Wuxi Matemity and Child Health \\ Care Hospital of Nanjing Medical University, Wuxi, China and ${ }^{5}$ Center of Clinical Reproductive Medicine, The \\ Affiliated Changzhou Matemity and Child Health Care Hospital of Nanjing Medical University, Changzhou, China
}

Correspondence should be addressed to X Huang or H Li or B Zheng; Email: bbhxy@njmu.edu.cn or hongliszivf@163.com ormansnoopy@163.com

*(B Zheng, J Yu and Y Guo contributed equally to this work)

\begin{abstract}
The cellular nucleic acid-binding protein (CNBP), also known as zinc finger protein 9, is a highly conserved zinc finger protein that is strikingly conserved among vertebrates. Data collected from lower vertebrates showed that CNBP is expressed at high levels and distributed in the testes during spermatogenesis. However, the location and function of CNBP in mammalian testes are not well known. Here, by neonatal mouse testis culture and spermatogonial stem cells (SSC) culture methods, we studied the effect of CNBP knockdown on neonatal testicular development. Our results revealed that CNBP was mainly located in the early germ cells and Sertoli cells. Knockdown of CNBP using morpholino in neonatal testis culture caused disruption of seminiferous tubules, mislocation of Sertoli cells and loss of germ cells, which were associated with the aberrant Wnt/B-catenin pathway activation. However, knockdown of CNBP in SSC culture did not affect the survival of germ cells. In conclusion, our study suggests that CNBP could maintain testicular development by inhibiting the Wnt/ $\beta$-catenin pathway, particularly by influencing Sertoli cells.

Reproduction (2018) 156 59-69
\end{abstract}

\section{Introduction}

The seminiferous epithelium of the neonatal mouse testis contains two distinct cell types, gonocytes and Sertoli cells. The first wave of spermatogenesis in the neonatal mouse testis is characterized by the differentiation of gonocytes found in the center of the seminiferous tubules. By 6 days after birth, the germ cells are attached to the basement membrane, and they differentiate into spermatogonial stem cells (SSCs). Next, SSCs are triggered to produce an optimal number of spermatogonia, which give rise to primary spermatocytes, and also to maintain a pool of stem cells. Then, the primary spermatocytes undergo a lengthy meiotic prophase, followed by the first meiotic division resulting in the formation of two secondary spermatocytes, each undergoing the second meiotic phase to produce four haploid round spermatids. Finally, haploid spermatids undergo spermiogenesis, which is a complex morphological transformation in sperm development (McLean et al. 2003, Kolasa et al.
2012). During early postnatal development, Sertoli cells continue to proliferate, differentiate and mature as nurse-like cells. Successful spermatogenesis relies on these early events in Sertoli cells because abnormal Sertoli cell proliferation or differentiation can disrupt fertility (Sharpe et al. 2003, Chojnacka et al. 2016).

CNBP encodes a $19 \mathrm{kD}$ protein containing seven tandem zinc finger repeats of 14 amino acid residues (Cys-X2-Cys-X4-His-X4-Cys). The strikingly conserved amino acid sequence of CNBP suggests that it plays an essential biological role across different species (Shimizu et al. 2003). Previous reports have implicated CNBP in two apparently unrelated biological processes: embryogenesis of craniofacial structures and myotonic dystrophy type 2 or proximal myotonic myopathy, which is a disorder affecting humans (Calcaterra et al. 2010). Furthermore, studies have shown that CNBP acts as a nucleic acid chaperone with both single-strand DNAand RNA-binding activity (Michelotti et al. 1995, Yasuda et al. 1995, Armas et al. 2008b). For example, CNBP 
activates c-myc transcription both in vivo and in vitro (Shimizu et al. 2003, Armas et al. 2008a); data from Armas et al. and Margarit et al. indicate that CNBP modulates the transcription of Wnt signaling pathway components (Wnt5b, Cdk14, Ptk7 and Tcf7/2) (Armas et al. 2013, Margarit et al. 2014). Wnt signaling is a highly conserved cell-to-cell communication mechanism, including canonical and noncanonical branches. Canonical Wnt signaling is also referred to as the $\mathrm{Wnt} / \beta$-catenin pathway and is often implicated as a stem cell self-renewal mechanism (Clevers et al. 2014).

In gibel carp testis, CNBP is expressed at high levels in spermatogonia and somatic cells, relatively low level in spermatocytes, and it is not expressed at all in sperm (Liu et al. 2009b). However, the roles of CNBP during spermatogenesis remain elusive. In our previous studies, we successfully identified CNBP in fetal testes and in the proteome profile for neonatal mouse testis (Zheng et al. 2014a, 2015). Bioinformatics analysis in our studies revealed that CNBP was associated with testicular development. In the present study, we aimed to study the expression level and distribution of CNBP in mouse neonatal testis and study the effects of its knockdown in vitro.

\section{Materials and methods}

\section{Animals}

Pregnant CD1 mice were maintained in a controlled environment under a $12 / 12$-h light/darkness cycle at $20-22^{\circ} \mathrm{C}$ and $50-70 \%$ humidity with food and water available ad libitum. All mouse experiments were approved by the Ethics Committee of Nanjing Medical University (NO. 14030119).

\section{Total RNA extraction and Real-time PCR}

Total RNA was extracted with the RNeasy Plus Micro Kit (Qiagen), according to the manufacturer's instructions. In short, the tissue was homogenized and transferred to gDNA Eliminator spin column to remove the genomic DNA. Then, sample was transferred to the RNeasy MinElute spin column and washed by series ethanol and wash buffers. Total RNA was finally eluted by $14 \mu \mathrm{L}$ RNase-free water and reversetranscribed into cDNA with reverse transcriptase (TaKaRa, Dalian, China). Gene expression was quantified with SYBR Premix Ex Taq kit (TaKaRa) in an ABI 7300 real-time PCR instrument (Applied Biosystems), using the standard curve method with 18s rRNA as the control gene. Primer sequences (5'-3'): Cnbp-For: CGAGTCGCCTTCTTGGTTCT; Cnbp-Rev: ACAAACTGGAACCCTCTGCC; Wnt5b-For TCTCTTAGTGGCCCCAGGTT; Wnt-5bRev: GCTAGTGACCACCAGGAGTT; $18 s$ rRNA-For: GATCCATTGGAGGGCAAGTCT; $18 \mathrm{~s}$ rRNA-Rev: CCAAGATCCAACTACGAGCTTTTT. The relative concentration of the candidate gene expression was calculated using the formula $2^{-\Delta \Delta C T}$ (Pfaffl 2001) as described in the SYBR Green user manual.

\section{Western blot analysis}

Western blot analysis was performed as described previously, with minor modifications (Kang et al. 2015). Briefly, testis lysates were separated by electrophoresis and then transferred to PVDF membranes (GE Healthcare). The membranes were then blocked in 5\% non-fat milk and incubated overnight with primary antibodies against CNBP (1:2000, Proteintech, Chicago, IL, USA), $\beta$-tubulin (1:10000, Beyotime,Beijing, China) and $\beta$-catenin (1:2000, BD Sciences, Franklin Lakes, NJ, USA), washed and incubated at room temperature for $1 \mathrm{~h}$ with horseradish peroxidase-conjugated secondary antibodies (Thermo Scientific). The protein signals were then visualized by SuperSignal West Femto Chemiluminescent Substrate (Thermo Scientific), and the images were obtained by X-ray films (Kodak). Band intensities were calculated by using Image-Pro Plus Software.

\section{Immunofluorescence}

Testis tissues were obtained from mice at 0, 7, 14 and 28 days of age, and adult mice or explant tissues were fixed in $4 \%$ paraformaldehyde. The tissues were embedded in paraffin and then sectioned into $5 \mu \mathrm{m}$ thick sections. For immunofluorescence, the sections were deparaffinized and rehydrated in a graded ethanol series. After antigen retrieval in sodium citrate buffer, the sections were blocked with $5 \%$ BSA and incubated overnight at $4{ }^{\circ} \mathrm{C}$ with primary antibodies against CNBP (1:200, Proteintech), DDX4 (1:500, Abcam), STIM1 (1:200, BD Sciences), SOX9 (1:500, Millipore), WT1 (1:100, Abcam), GATA4 (1:100, Santa Cruz Biotechnology), DAX1 (1:200, Cell Signaling), Laminin (1:500, Abcam,), $3 \beta$-HSD (1:500, Santa Cruz), $\beta$-catenin (1:500, BD) and C-caspase3 (1:200, Cell Signaling). After washing the samples with phosphate-buffered saline-Tween, the sections were incubated with Alexa Fluor secondary antibodies (Thermo Scientific), and the cell nuclei were stained with $5 \mu \mathrm{g} / \mathrm{mL}$ DAPI (Beyotime) for $5 \mathrm{~min}$. Images were captured under a confocal laser microscope (Zeiss LSM710, Carl Zeiss). For SSCs immunofluorescence analysis, SSCs were cultured in a Millicell EZ slide (Millipore Corporation). Cells were fixed in $4 \%$ paraformaldehyde, blocked with 5\% BSA and incubated overnight at $4{ }^{\circ} \mathrm{C}$ with primary antibodies against $\beta$-catenin (1:500, BD Sciences), PLZF (1:500, Santa Cruz), GFR $\alpha-1$ (1:500, R\&D Systems, Minneapolis, MN, USA) and LIN28 (1:1000, Abcam). After washing the samples with phosphatebuffered saline, the sections were incubated with Alexa Fluorconjugated secondary antibodies (Thermo Scientific), and the cell nuclei were stained with $5 \mu \mathrm{g} / \mathrm{mL}$ DAPI (Beyotime) for $1 \mathrm{~min}$. All samples were observed under a confocal laser microscope (Zeiss LSM710, Carl Zeiss).

\section{Neonatal testis culture}

Testes from 5.5-day-old mice were decapsulated and gently cut into several $1-3 \mathrm{~mm}$ diameter pieces. Testis explants were cultured as previously reported (Sato et al. 2011). Briefly, $1.5 \%(\mathrm{w} / \mathrm{v})$ agarose gel stands $\left(10 \times 10 \times 5 \mathrm{~mm}^{3}\right.$ in size and placed in 24-well plates) were prepared 1 day 
before the testis culture and incubated with culture medium for more than $24 \mathrm{~h}$. The amount of medium was adjusted, so it would come up to half to four-fifth of the height of the agarose gel. The medium was changed twice a week. Then, 3-5 testes explants were placed on the medium/ air interface of each stand and incubated at $34^{\circ} \mathrm{C}$ and $5 \%$ $\mathrm{CO}_{2}$. The culture media contained $\alpha$-minimum essential medium, $10 \%$ knockout serum replacement, 1\% nonessential amino acid solution, $0.1 \% \beta$-mercaptoethanol and $1 \%$ penicillin-streptomycin solution. The explants were cultured for 9 days either with $15 \mu \mathrm{M}$ morpholino (Mo) or negative control Mo (Ctr). To verify the efficiency of Mo, testis explants were cultured for $48 \mathrm{~h}$ with $15 \mu \mathrm{M}$ Mo or Ctr. CNBP translation-blocking vivo-Mo (Oligo Sequence: TGAAGCATTCGTTGCTGCTCATAGC) and the control (Oligo Sequence: CCTCTTACCTCAGTTACAATTTATA) were purchased from Gene Tools LLC, Philomath, OR, USA. The CNBP Mo oligos can bind to the complementary RNA and then block translation initiation in the cytosol by targeting the $5^{\prime}$ UTR through the first 25 bases of coding sequence. XAV939 was obtained from Selleck (Houston, TX, USA) and used at a final concentration of $5 \mathrm{nM}$. For each experiment, at least eight testes explants were prepared in each group.

\section{Hematoxylin and eosin staining}

The testis explants were fixed in $4 \%$ paraformaldehyde, embedded in paraffin, sectioned at $5 \mu \mathrm{m}$, deparaffinized and stained with hematoxylin and eosin (H\&E). After dehydration through a series of graded ethanol baths and clearing in xylene, the samples were mounted with neutral balsam and viewed under a bright-field microscope (Axioskop 2 plus, Zeiss).

\section{SSC culture}

Long-term culture of mouse SSCs was established in accordance with a previous described protocol (Zhou et al. 2015). Briefly, testis from 2-day neonatal mice was digested with $1 \mathrm{mg} / \mathrm{mL}$ collagenase (type IV; Sigma-Aldrich) for $15 \mathrm{~min}$, followed by $0.25 \%$ trypsin EDTA (Invitrogen) with $1.4 \mathrm{mg} / \mathrm{mL}$ DNase (Sigma-Aldrich) for $10 \mathrm{~min}$. Dissociated testicular cells were cultured overnight on a $0.2 \%(\mathrm{w} / \mathrm{v})$ gelatin-coated tissue culture plate $\left(2 \times 10^{5}\right.$ cells $\left./ 3.8 \mathrm{~cm}^{2}\right)$. The plates were washed twice with PBS before use. Floating cells were passaged to secondary plates. These cells were then passaged two or three times before they were transferred to medium containing mouse embryo fibroblasts. The cells were maintained at $37^{\circ} \mathrm{C}$ in air containing $5 \%$ carbon dioxide. SSCs were cultured for 5-6 days, either with $15 \mu \mathrm{M}$ Mo or Ctr.

\section{Statistical analysis}

Experiments were repeated at least three times. The data were evaluated for statistical differences using Student $t$-test and one-way ANOVA by GraphPad software with $* P<0.05$, $* * P<0.01$ and $* * * P<0.001$.

\section{Results}

\section{CNBP expression pattern during spermatogenesis in mice}

First, we used real-time PCR to detect the Cnbp mRNA level. The results revealed high Cnbp expression at 0and 7-day testes, with a dramatic decline from 14 days onward, and very low Cnbp expression in adult testes (Fig. 1A). Protein expression was analyzed by western blot analysis and the results were consistent with the mRNA expression pattern (Fig. 1B and C). We then performed immunostaining to analyze the distribution of CNBP in the testis at different stages of development. As shown in Fig. 1D, CNBP was mainly located in the seminiferous epithelium of 0 - and 7-day testes. In 14-day testes, there was a relatively weak signal for CNBP in spermatocytes, whereas the fluorescence signal was very low from 28 days. Moreover, by immunostaining with germ cell marker, DDX4 (also known as VASA or MVH) (Kuramochi-Miyagawa et al. 2010) and Sertoli cell marker, STIM1 (Zheng et al. 2015), we found CNBP was located in both germ cells and Sertoli cells (Fig. 1E). Taken together, the expression data indicate that CNBP is associated with neonatal testicular maturation.

\section{CNBP is essential for germ cell survival}

To explore the function of CNBP in neonatal testis, we used Mo oligo to knockdown CNBP and successfully suppressed CNBP translation in vitro. Western blot analysis showed $56.8 \%$ knockdown efficiency of CNBP by Mo in vitro $(P=0.0084)$ (Fig. $2 \mathrm{~A}$ and $\mathrm{B})$. The cultured testis explants incubated with Mo for 9 days were morphologically examined by H\&E staining, and the results revealed a large percentage of cells in the Mo group had undergone apoptosis (Fig. 2C). The apoptotic signals were further verified using the TUNEL assay (Fig. 2D) and immunostaining for cleaved caspase-3 (Fig. 2E). The TUNEL and cleaved caspase-3-positive cells showed 3.94-fold and 3.8-fold increase in Mo testes respectively, compared with $\mathrm{Ctr}$ $(P=0.00064$ and 0.00058 respectively). (Fig. 2D and E). In agreement with this finding, we observed a $64.1 \%$ reduction of DDX4-labeled germ cells in Mo testes $(P=0.000297)$ (Fig. 2F). Furthermore, after incubation with Mo for 20 days, which corresponds to the round spermatid stage, the Mo group exhibited Sertoli cellonly phenotype, while the $\mathrm{Ctr}$ group showed normal development of round spermatids (Fig. 2G and H). The above data indicate that CNBP is associated with germ cell survival.

\section{CNBP inhibition affects Sertoli cell orientation but not identification}

As CNBP is distributed in both germ cells and Sertoli cells, we attempted to compare the orientation and 


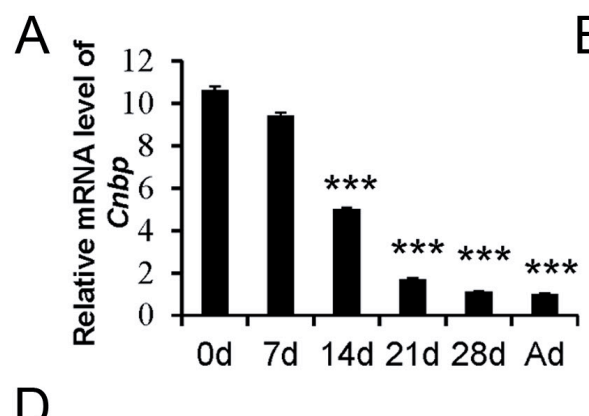

B
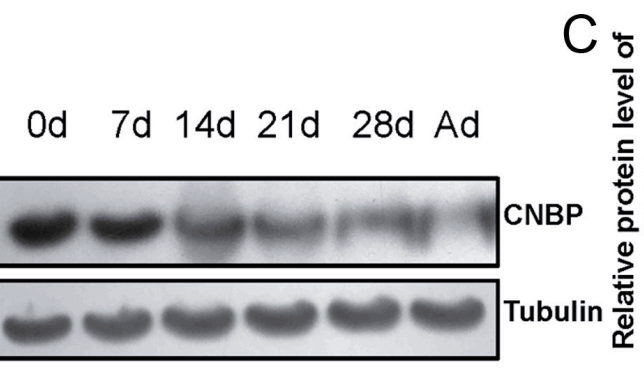

DAPI/ CNBP
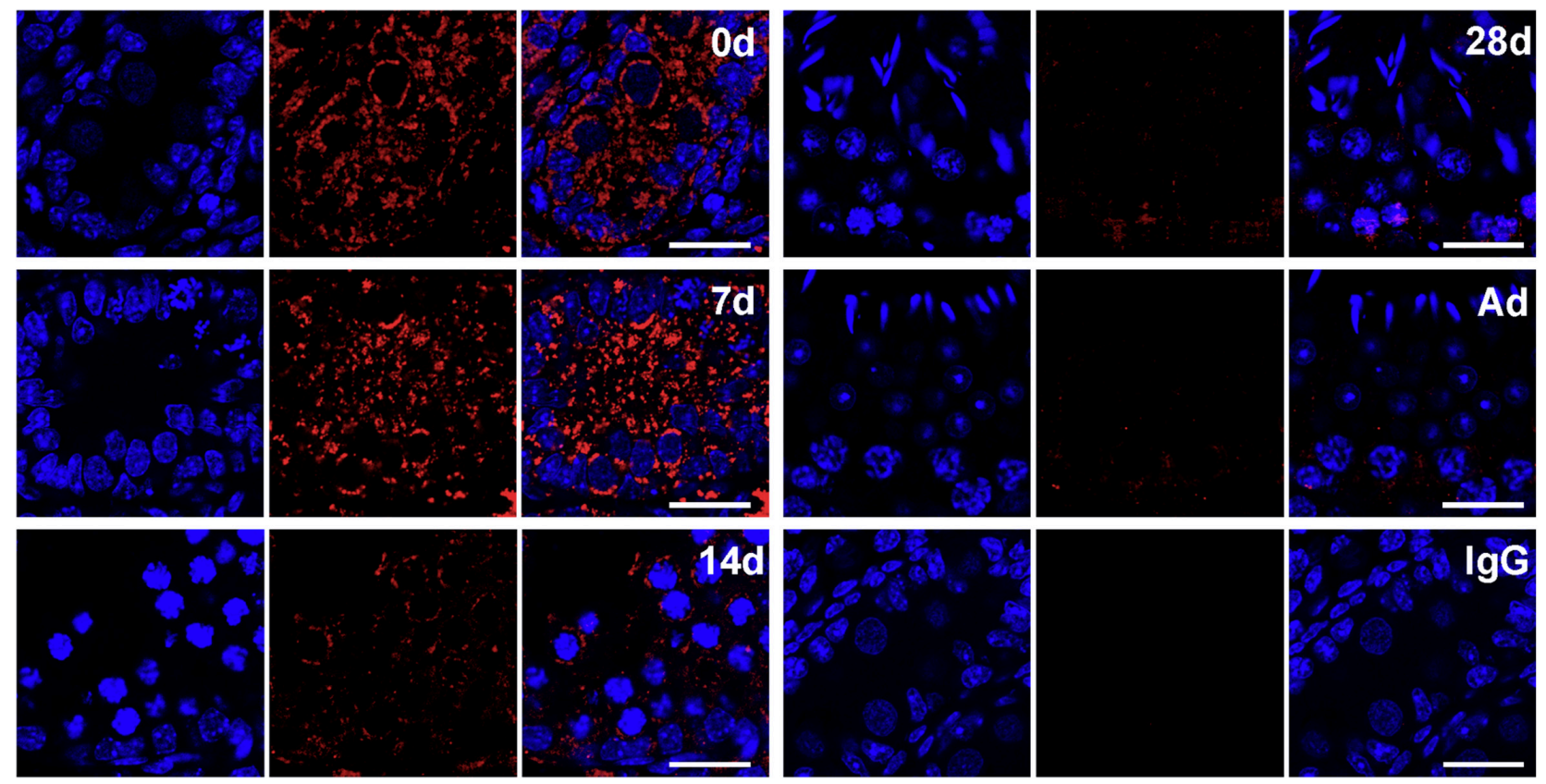

$E$

DAPI/DDX4/ CNBP

DAPI/STIM1/ CNBP
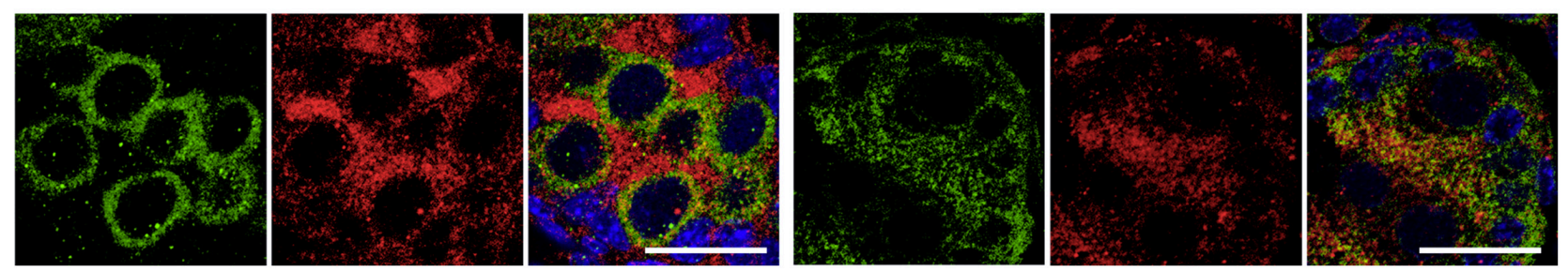

Figure 1 Expression and distribution of CNBP during spermatogenesis. (A) Relative Cnbp mRNA level in mouse testis at different ages. (B) Western blot analysis of the CNBP protein level in mouse testis at different ages. (C) Quantification of (B). (D) Representative images of CNBP immunostaining in mouse testis at different ages. Confocal immunofluorescence analysis showed that CNBP was located in the cytoplasm. IgG was used as the negative control. (E) Co-immunostaining of CNBP and DDX4 or STIM1 in 0-day testes. CNBP was partly co-located with DDX4 and STIM1 respectively. Scale bar: $20 \mu \mathrm{m}$. CNBP, cellular nucleic acid-binding protein.

identification of Sertoli cells in the Mo and Ctr group. We evaluated the expression levels of several Sertoli cell markers in cultured testes. Sertoli cells in the Ctr group were surrounded with the basal membrane consistently (Fig. 3A, yellow arrowheads), while Sertoli cells in the Mo group departed from the basal membrane and translocated into the adluminal compartment (Fig. 3A,
B, C and D, dotted circles). The number of Sertoli cells did not appear to differ between the two treatment groups (Fig. 3A, B, C and D). Although CNBP-deficient testicular tissue had fewer germ cells and mis-localized Sertoli cells, they could still be identified using the SOX9 (Fig. 3A), WT1 (Fig. 3B), GATA4 (Fig. 3C) and DAX1 (Fig. 3D) markers. 

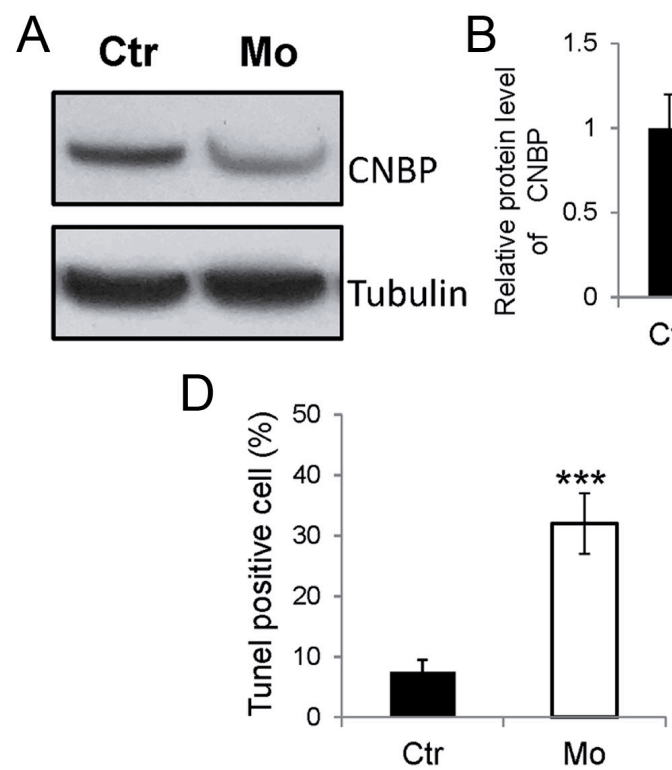

E

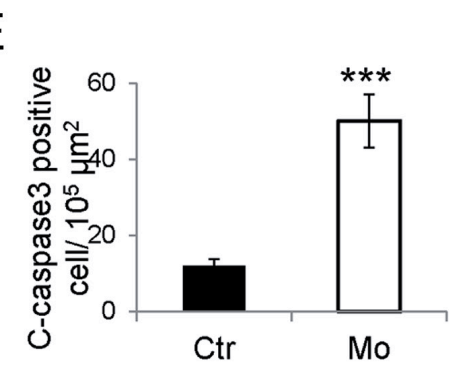

$\mathrm{F}$

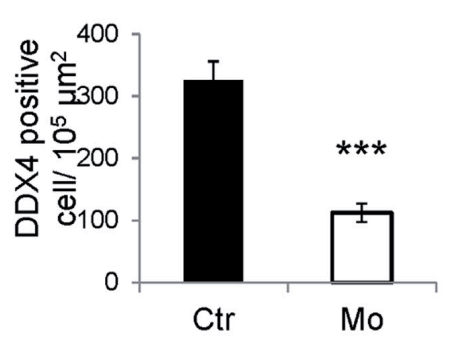

C

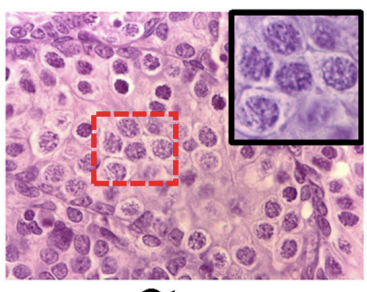

Ctr

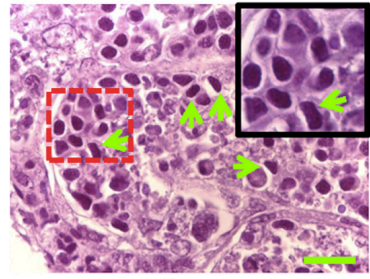

Mo

Tunel/ DAPI

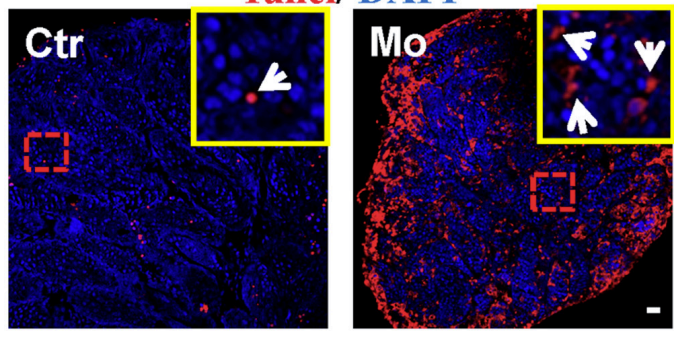

\section{C-caspase3/ DAPI}
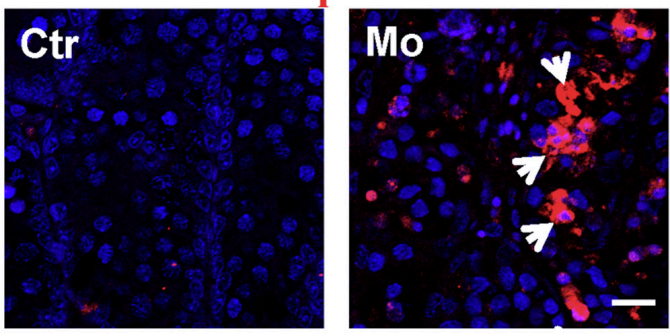

DDX4/ DAPI
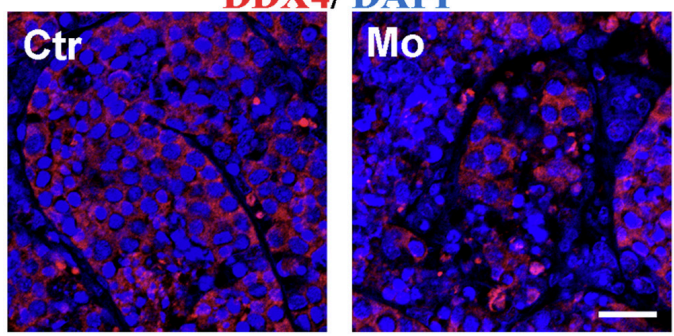

G
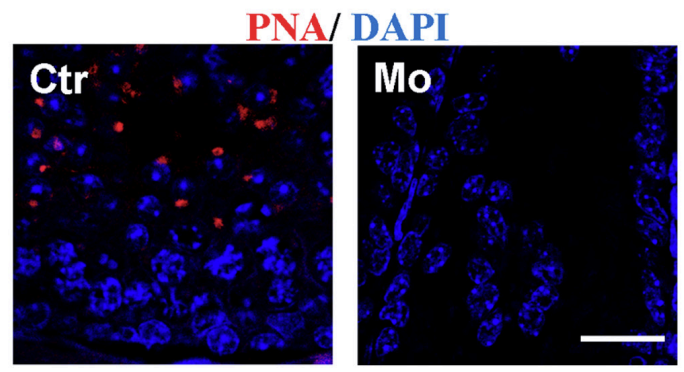

$\mathrm{H}$

DDX4/ DAPI
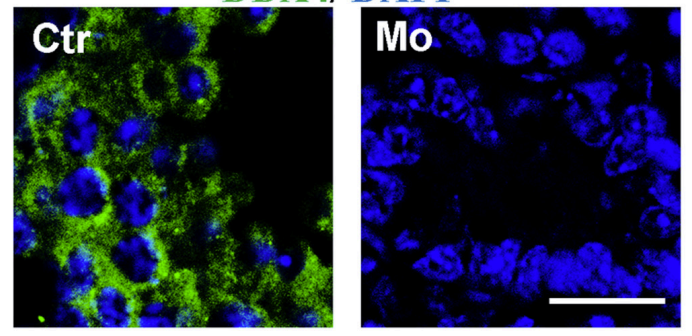

Figure 2 CNBP is required for germ cell survival. (A) Western blot analysis showed high knockdown efficiency of CNBP by Mo. (B) Quantification of (A). (C) Representative images of H\&E staining of the Ctr and Mo testes. Green arrows indicate apoptotic cells. (D) Representative images of TUNEL assay of Ctr and Mo testes. White arrows indicate apoptotic cells. (E) Representative images of cleaved caspase-3 immunostaining from Ctr and Mo testes. White arrows indicate apoptotic cells. (F) Representative images of DDX4 immunostaining in $\mathrm{Ctr}$ and Mo testes. (G) Representative images of PNA staining from $\mathrm{Ctr}$ and Mo testes. PNA indicates the acrosome of spermatids. (H) Representative images of DDX4 immunostaining in Ctr and Mo testes. DDX4-labeled germ cells were undetectable in testes incubating with Mo for 20 days. Scale bar: $20 \mu \mathrm{m}$. CNBP, cellular nucleic acid-binding protein; Ctr, negative control Mo; H\&E, hematoxylin and eosin; Mo, morpholino. 
A

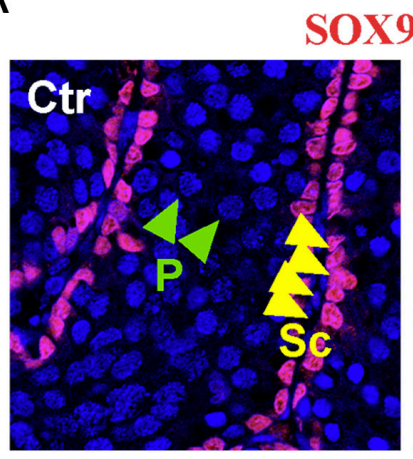

B

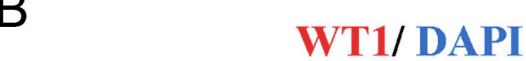

C

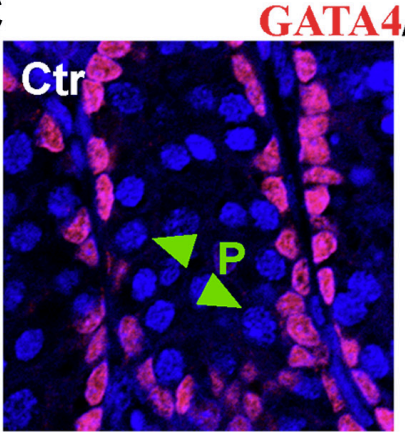

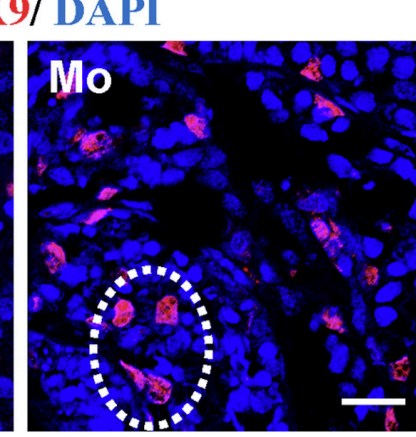
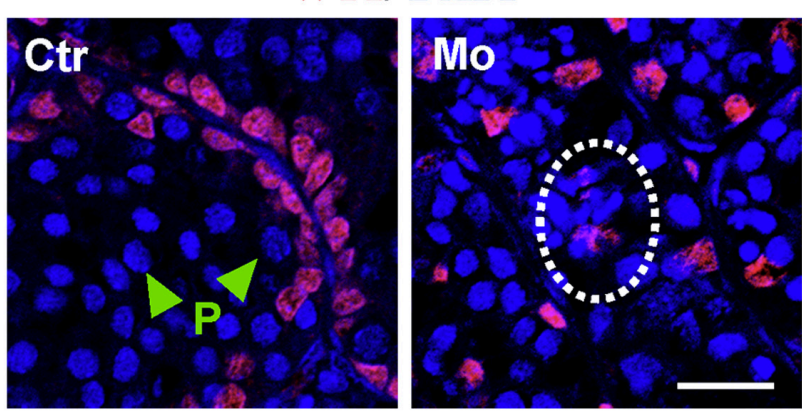

D

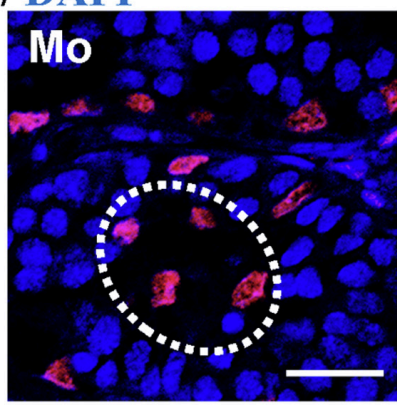

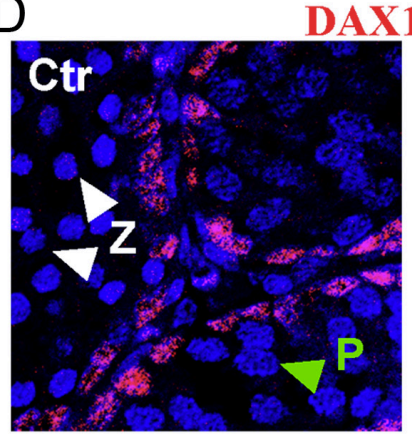

Figure 3 CNBP is required for Sertoli cells organization, but not identification. (A, B, C and D) Representative images of SOX9, WT1, GATA4 and DAX1 immunostaining in Ctr and Mo testes respectively. Mis-located Sertoli cells were indicated by dotted circles. Scale bar: $20 \mu \mathrm{m}$. CNBP, cellular nucleic acid-binding protein; Ctr, negative control Mo; Mo, morpholino; P, pachytene spermatocyte; Sc, Sertoli cells; Z, zygotene spermatocyte.

\section{Seminiferous tubules are disturbed in CNBP-deficient testes}

Findings from us and other research groups have shown that Sertoli cells act as an organizing center in testicular cord formation (Svingen \& Koopman 2013, Zheng et al. 2015). To detect the integrity of seminiferous tubules after CNBP knockdown, we labeled the basal laminae of testes by laminin, and the results revealed that the seminiferous tubules were severely disrupted in the Mo group (Fig. 4A). Gao et al. showed that the absence of normal tubular architecture in testes can lead to the expansion of Leydig cells (Gao et al. 2006). To test this, we performed immunostaining of Leydig cells using $3 \beta$ HSD, and the results implied 2.3-fold increase of Leydig cells in the Mo interstitium ( $P=0.00097)$ (Fig. 4B).

\section{Aberrant activation of the $W N T / \beta$-catenin pathway in CNBP-deficient testes}

The WNT/B-catenin pathway is a fundamental signaling pathway that regulates cell fate, cell polarity and homeostasis. Activation of the WNT/ $\beta$-catenin pathway resulted in the translocation of $\beta$-catenin to the nucleus and formation of a heterodimeric complex with the Tcf/Lef family of DNA-binding proteins, thus regulating the transcription of downstream target genes
(Loh et al. 2016). We found a 5.3-fold increase in the Wnt5b mRNA level in Mo compared with the Ctr group $(P=0.00003)$ (Fig. 5A). In addition, western blot data also indicated 2.8 -fold elevation of $\beta$-catenin expression

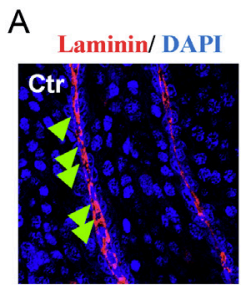

B
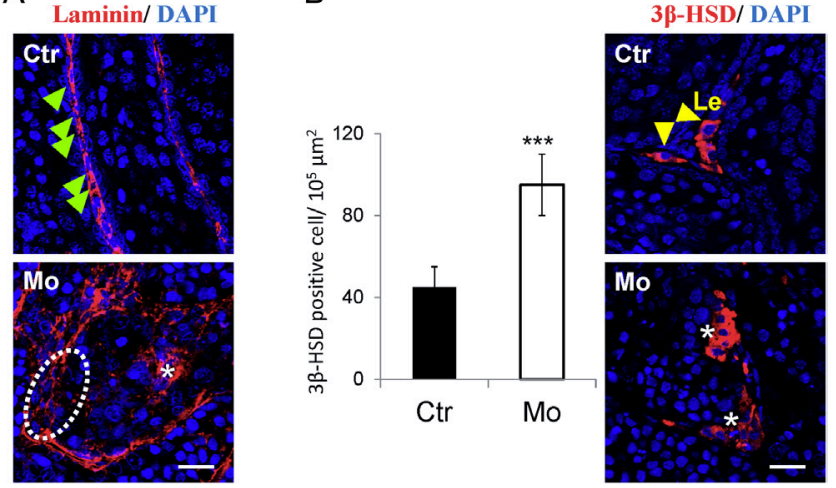

Figure 4 Knockdown of CNBP disrupts seminiferous tubules. (A) Representative images of laminin immunostaining in Ctr and Mo testes. In control testes, green arrowheads indicate the well-formed laminin. In Mo testes, asterisk indicates the disorganized laminin; dotted circle indicates laminin loss. (B) Representative images of $3 \beta$-HSD immunostaining in Ctr and Mo testes. Asterisks indicate the expansion of Leydig cells. Scale bar: $20 \mu \mathrm{m}$. CNBP, cellular nucleic acid-binding protein; Ctr, negative control Mo; Le, Leydig cells; Mo, morpholino. 
A

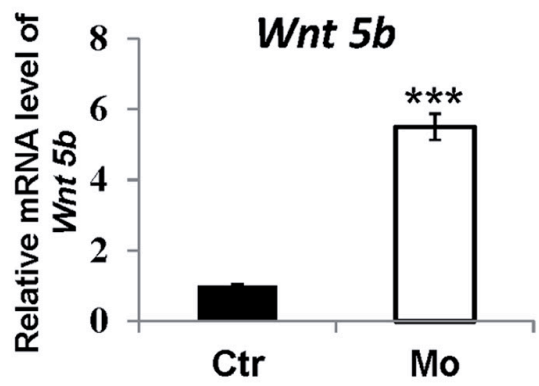

D
B

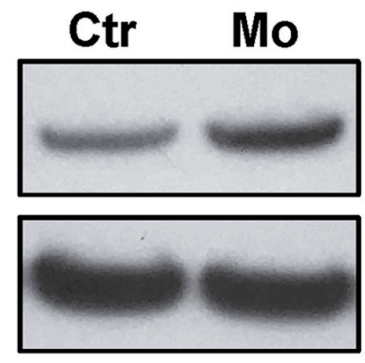

C

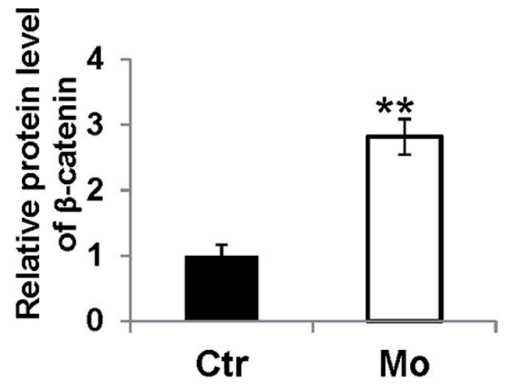

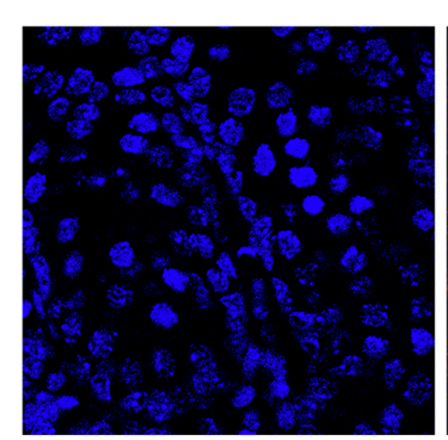

DAPI/ $\beta$-catenin
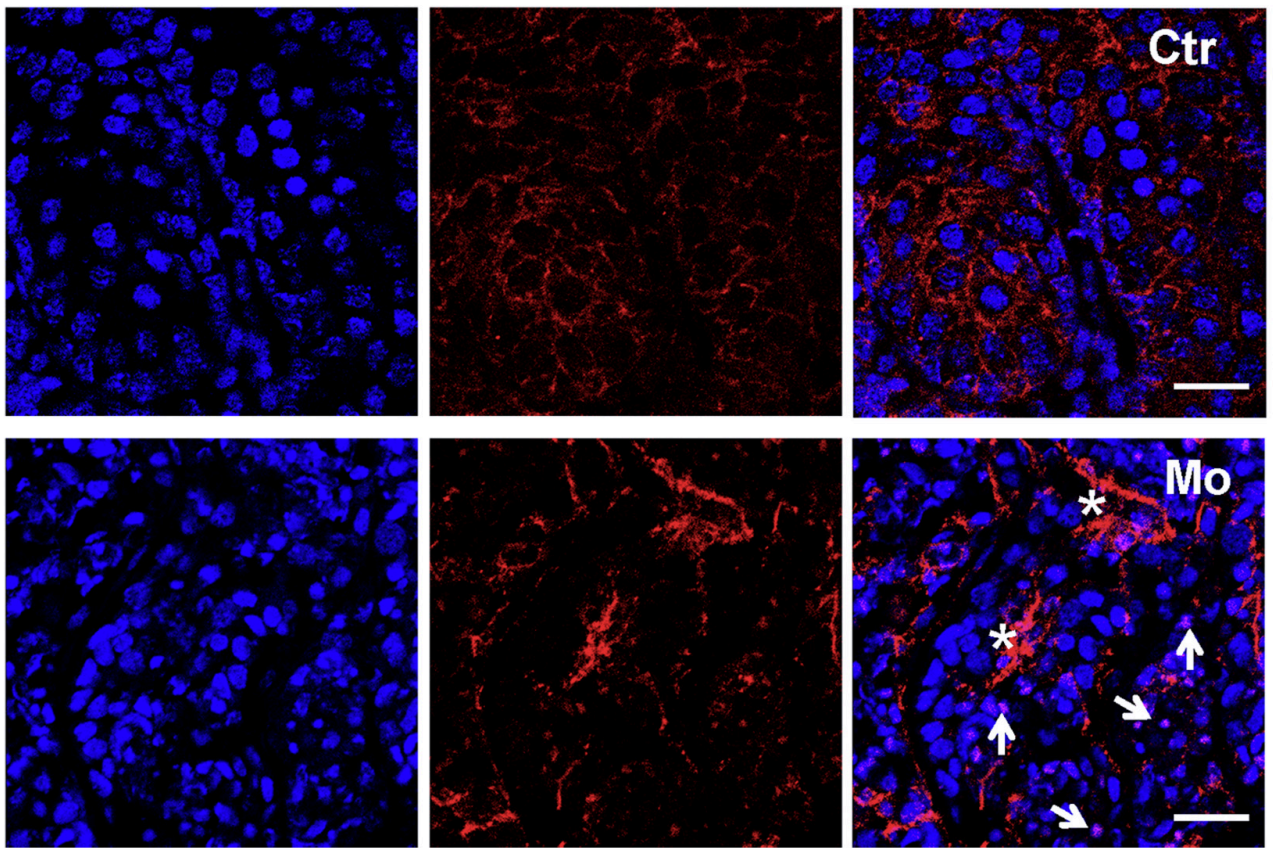

Figure 5 CNBP inhibition leads to aberrant activation of the WNT/ $\beta$-catenin pathway. (A) Relative mRNA level of Wnt5b in Ctr and Mo testes. (B) Western blot analysis of $\beta$-catenin expression in Ctr and Mo testes. (C) Quantification of (B). (D) Representative images of $\beta$-catenin immunostaining in $\mathrm{Ctr}$ and Mo testes. Asterisks indicate the aberrant increase in cytoplasmic $\beta$-catenin in Mo testes; white arrows indicate the translocation of $\beta$-catenin into the nucleus in Mo testes. Scale bar: $20 \mu \mathrm{m}$. CNBP, cellular nucleic acid-binding protein; Ctr, negative control Mo; Mo, morpholino.

in Mo $(P=0.00285)$ (Fig. 5B and C). Furthermore, immunostaining of $\beta$-catenin in Mo-treated testes showed significant accumulation and translocation of $\beta$-catenin into the nucleus (Fig. 5D). Together, these data demonstrate that the $\mathrm{WNT} / \beta$-catenin pathway is activated in CNBP-deficient testes.

\section{Inhibition of the $W N T / \beta$-catenin pathway can partly rescue the altered phenotypes}

To verify whether the aberrant activation of the WNT/ $\beta$ catenin pathway was a crucial contributor to the severe phenotypes observed in this study, we treated cultured testicular cells with the WNT/ $\beta$-catenin pathway inhibitor XAV939 (Lien et al. 2014). Both western blot
(Fig. 6A and B) and immunostaining (Fig. 6C) results exhibited that XAV939 inhibited the WNT/B-catenin pathway with high efficiency. Western blot analysis of $\beta$-catenin expression showed $32.3 \%$ reduce in the XAV939-treated Mo testes, compared with Mo (Ctr vs Mo, $P=0.00094 ;$ Mo vs Mo+XAV939, $P=0.0071$ ) (Fig. 6B). The defects in testicular morphology were partly rescued after treatment with XAV939 (Fig. 6D), and the number of germ cells in the XAV939-treated Mo group showed 2.36-fold increase compared with Mo group (Ctr vs Mo, $P=0.000745$; Mo vs Mo+XAV939, $P=0.0039$ ) (Fig. 6E), while apoptotic cells showed $46.5 \%$ reduce after treatment with XAV939 (Ctr vs Mo, $P=0.000228 ;$ Mo vs Mo+XAV939, $P=0.014$.) (Fig. 6F). 
A

C
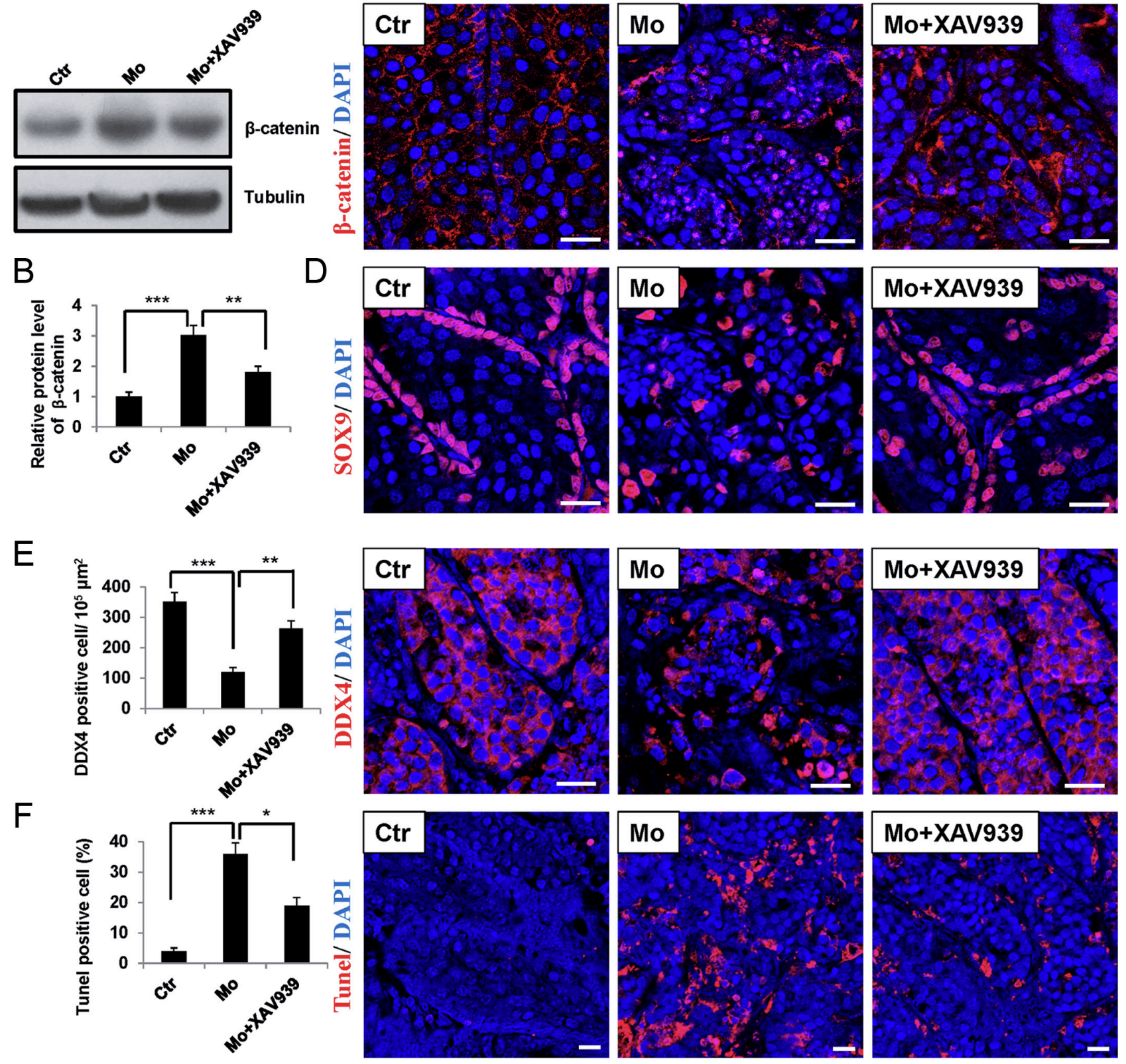

Figure 6 The WNT/ $\beta$-catenin pathway inhibitor can partly rescue the altered phenotypes. (A) Western blot analysis of $\beta$-catenin expression in Ctr, Mo and rescue testes. (B) Quantification of (A). (C) Representative images of $\beta$-catenin immunostaining in Ctr, Mo and rescue (Mo+XAV939) testes. XAV939 inhibited the WNT/ $\beta$-catenin pathway with high efficiency. (D) Representative images of SOX9 immunostaining in Ctr, Mo and rescue testes. Testicular morphology was partly rescued after treatment with XAV939. (E) Representative images of DDX4 immunostaining in Ctr, Mo and rescue testes. The loss of germ cells in the Mo was reversed after treatment with XAV939. (F) Representative images of TUNEL assay in Ctr, Mo and rescue testes. Scale bar: $20 \mu \mathrm{m}$. Ctr, negative control Mo; Mo, morpholino.

\section{Deficiency of CNBP does not influence apoptosis and $\beta$-catenin location in SSCS}

To observe whether CNBP knockdown was a direct or indirect cause of the observed germ cell apoptosis, we incubated SSC culture with Mo. As shown in Fig. 7A, there was no difference in the apoptosis level between the Mo (6.1\%) and $\mathrm{Ctr}(5.5 \%)$ groups $(P=0.56)$. $\beta$-Catenin was still located in the cytoplasm after Mo treatment. We did not observe translocation of $\beta$-catenin to the nucleus and immunostaining with SSC markers indicated that the SSC colonies showed normal growth (Fig. 7B, C and D). 
A

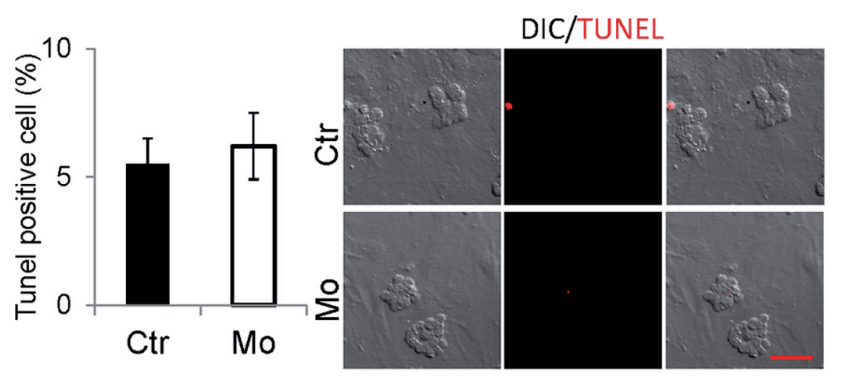

C
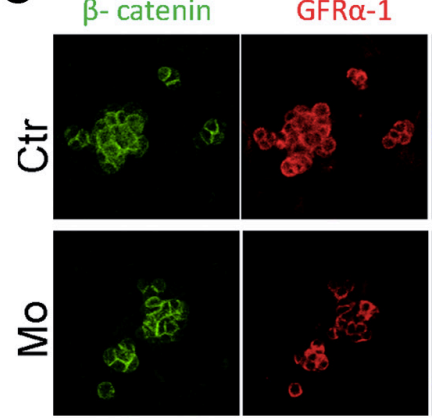

DAPI
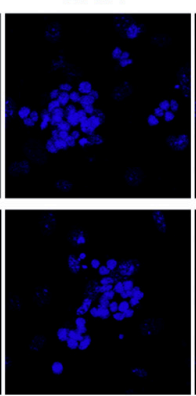

B

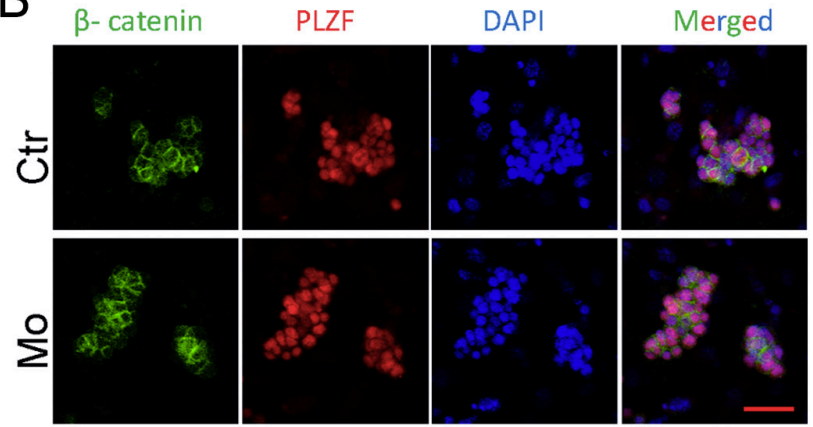

Figure 7 CNBP knockdown in SSC culture did not influence apoptosis of SSCs and distribution of $\beta$-catenin. (A) Representative images of TUNEL assay in Ctr and Mo SSCs. (B) Representative images of $\beta$-catenin and PLZF immunostaining in Ctr and Mo SSCs. (C) Representative images of $\beta$-catenin and GFR $\alpha-1$ immunostaining in Ctr and Mo SSCs. (D) Representative images of $\beta$-catenin and LIN28 immunostaining in Ctr and Mo SSCs. Scale bar: $50 \mu \mathrm{m}$. CNBP, cellular nucleic acid-binding protein; Ctr, negative control Mo; Mo, morpholino; SSC, spermatogonial stem cell.

\section{Discussion}

This is the first study to report the distribution and function of CNBP in mammalian testes. We found that CNBP was primarily located in the early germ cells and Sertoli cells. Knockdown of CNBP by Mo in neonatal testis culture resulted in disrupted seminiferous tubule integrity, reduced germ cell survival and WNT/ $\beta$-catenin pathway activation. Furthermore, treatment with the WNT/B-catenin pathway inhibitor XAV939 resulted in partial rescue of these phenotypes. However, Mo treatment to inhibit CNBP in SSC culture did not affect the apoptosis of germ cells. There were no differences in the SSC colony size, and the distribution of SSC markers in both the Mo and Ctr groups appeared normal. Overall, our results demonstrated that germ cell apoptosis in neonatal testis culture might be an indirect result of CNBP inhibition and that CNBP could regulate neonatal testicular development, particularly by influencing Sertoli cells via the WNT/ $\beta$-catenin pathway.

The WNT/ $\beta$-catenin pathway enables cells to communicate with each other and to coordinate various cellular processes such as cell proliferation, differentiation, survival, apoptosis, migration and cell fate (Logan \& Nusse 2004). A hallmark of the WNT/ $\beta$ catenin pathway is the nuclear localization of $\beta$-catenin (Brown 2005). Several studies have recently established the contribution of regulated WNT/ $\beta$-catenin signaling within Sertoli cells to male fertility via mouse models that activate or block WNT/ $\beta$-catenin signaling. Constitutive activation of $\beta$-catenin in Sertoli cells that begins during embryogenesis under the control of $\mathrm{AMH}$-Cre, which expresses Cre only in Sertoli cells after embryonic day 13.5 , results in disrupted tubular structure, disorganized Sertoli cells, loss of germ cells and hyperplasia of Leydig cells in the embryonic stage and complete absence of spermatogenesis in adults (Chang et al. 2008). Similarly, postnatal expression of activated $\beta$-catenin in Sertoli cells under the control of AMHR2-Cre, which expresses Cre in both Sertoli and Leydig cells postnatally, results in progressive atrophy of the tubules starting at 5 weeks of age, associated with germ cells loss and hyperplasia of the testicular interstitial cells (Boyer et al. 2008). In contrast, deletion of $\beta$-catenin in Sertoli cells causes no phenotypic defects at the embryonic or postnatal stages (Chang et al. 2008, Liu et al. 2009a). These studies collectively indicate that the constitutive activation of the WNT/ $\beta$-catenin pathway in Sertoli cells causes spermatogenic defects, while deficiency of $\beta$-catenin does not affect Sertoli cells to support germ cell development.

Consistent with these previously published results from embryonic stage and puberty, our present study showed 
severe phenotypes, including disrupted seminiferous tubules, disorganized Sertoli cells, loss of germ cells and hyperplasia of Leydig cells, in association with aberrant activation of the WNT/ $\beta$-catenin pathway in CNBPdeficient neonatal testes. Moreover, treatment with the WNT/ $\beta$-catenin pathway inhibitor XAV939 resulted in an apparent rescue of phenotypes. Furthermore, no translocation of $\beta$-catenin to the nucleus was observed in SSC culture. Based on these data, we concluded that the WNT/ $\beta$-catenin pathway is one of the crucial targets of CNBP in Sertoli cells and that it plays important roles in neonatal testicular development in mice.

Chen et al. previously showed that the Cnbp-null mutation is an embryonically lethal mutation in mice due to severe forebrain truncation and facial anomalies (Chen et al. 2003) caused in mice lacking this gene; therefore, a mutant mouse model is difficult to use in this case to study the role of CNBP in the testes. In our previous proteomic analysis, we identified CNBP and found that it might play a role during testes development (Zheng et al. 2014a, 2015). In the present study, we used neonatal mouse testis culture and SSC culture to observe the function of CNBP during spermatogenesis. Since Sato et al. first obtained functional sperm in cultured neonatal mouse testes (Sato et al. 2011), this culture method has been widely used as a platform for the mechanistic understanding of spermatogenesis by several researchers (Chen et al. 2014, Zheng et al. 2014b, 2015). As for SSC culture, it is also a stable platform for exploring the development of spermatogonial stem cells and molecular mechanisms of spermatogenesis (Zhou et al. 2015, Kanatsu-Shinohara et al. 2016, Helsel et al. 2017, Wang et al. 2017). However, future studies investigating the detailed molecular mechanism of CNBP in testes using conditional knockout mouse models are warranted.

\section{Declaration of interest}

The authors declare that there is no conflict of interest that could be perceived as prejudicing the impartiality of the research reported.

\section{Funding}

This work was supported by the Suzhou Key Medical Center (SZZX201505), Jiangsu Provincial Medical Innovation Team (CXTDB2017013), Suzhou Introduced Project of Clinical Medical Expert Team (SZYJTD201708), Key Research Fund for Zhenjiang Social Development (SH2016028), Key Research Fund for Zhenjiang Health Science and Technology (SHW2016001), National Natural Science Foundation of China (31701298), Natural Science Foundation of Jiangsu Province (20170562) and Open Fund of State Key Laboratory of Reproductive Medicine of Nanjing Medical University (SKLRM-KA201603 and SKLRM-KA201704).

\section{References}

Armas $\mathrm{P}$, Aguero TH, Borgognone $\mathrm{M}$, Aybar MJ \& Calcaterra NB 2008a Dissecting CNBP, a zinc-finger protein required for neural crest development, in its structural and functional domains. Journal of Molecular Biology 382 1043-1056. (https://doi.org/10.1016/j. jmb.2008.07.079)

Armas P, Nasif S \& Calcaterra NB 2008b Cellular nucleic acid binding protein binds G-rich single-stranded nucleic acids and may function as a nucleic acid chaperone. Journal of Cellular Biochemistry 103 1013-1036. (https://doi.org/10.1002/jcb.21474)

Armas P, Margarit E, Mouguelar VS, Allende ML \& Calcaterra NB 2013 Beyond the binding site: in vivo identification of tbx2, smarca5 and wnt5b as molecular targets of CNBP during embryonic development. PLOS ONE 8 e63234. (https://doi.org/10.1371/journal.pone.0063234)

Boyer A, Hermo L, Paquet M, Robaire B \& Boerboom D 2008 Seminiferous tubule degeneration and infertility in mice with sustained activation of WNT/CTNNB1 signaling in sertoli cells. Biology of Reproduction 79 475-485. (https://doi.org/10.1095/biolreprod.108.068627)

Brown AM 2005 Canonical Wnt signaling: high-throughput RNAi widens the path. Genome Biology 6 231. (https://doi.org/10.1186/gb-2005-69-231)

Calcaterra NB, Armas P, Weiner AM \& Borgognone M 2010 CNBP: a multifunctional nucleic acid chaperone involved in cell death and proliferation control. IUBMB Life 62 707-714. (https://doi.org/10.1002/ iub.379)

Chang H, Gao F, Guillou F, Taketo MM, Huff V \& Behringer RR 2008 Wt1 negatively regulates beta-catenin signaling during testis development. Development 135 1875-1885. (https://doi.org/10.1242/dev.018572)

Chen W, Liang Y, Deng W, Shimizu K, Ashique AM, Li E \& Li YP 2003 The zinc-finger protein $\mathrm{CNBP}$ is required for forebrain formation in the mouse. Development 130 1367-1379. (https://doi.org/10.1242/ dev.00349)

Chen M, Wang X, Wang Y, Zhang L, Xu B, Lv L, Cui X, Li W \& Gao F 2014 Wt1 is involved in leydig cell steroid hormone biosynthesis by regulating paracrine factor expression in mice. Biology of Reproduction 90 71. (https://doi.org/10.1095/biolreprod.113.114702)

Chojnacka K, Zarzycka M \& Mruk DD 2016 Biology of the sertoli cell in the fetal, pubertal, and adult mammalian testis. Results and Problems in Cell Differentiation 58 225-251. (https://doi.org/10.1007/978-3-31931973-5_9)

Clevers H, Loh KM \& Nusse R 2014 Stem cell signaling. An integral program for tissue renewal and regeneration: Wnt signaling and stem cell control. Science 346 1248012. (https://doi.org/10.1126/science.1248012)

Gao F, Maiti S, Alam N, Zhang Z, Deng JM, Behringer RR, Lecureuil C, Guillou F \& Huff V 2006 The Wilms tumor gene, Wt1, is required for Sox9 expression and maintenance of tubular architecture in the developing testis. PNAS 103 11987-11992. (https://doi.org/10.1073/ pnas.0600994103)

Helsel AR, Yang QE, Oatley MJ, Lord T, Sablitzky F \& Oatley JM 2017 ID4 levels dictate the stem cell state in mouse spermatogonia. Development 144 624-634. (https://doi.org/10.1242/dev.146928)

Kanatsu-Shinohara M, Tanaka T, Ogonuki N, Ogura A, Morimoto $\mathbf{H}$, Cheng PF, Eisenman RN, Trumpp A \& Shinohara T 2016 Myc/Mycnmediated glycolysis enhances mouse spermatogonial stem cell self-renewal. Genes and Development 30 2637-2648. (https://doi. org/10.1101/gad.287045.116)

Kang Y, Zheng B, Shen B, Chen Y, Wang L, Wang J, Niu Y, Cui Y, Zhou J, Wang $\mathbf{H}$ et al. 2015 CRISPR/Cas9-mediated Dax1 knockout in the monkey recapitulates human AHC-HH. Human Molecular Genetics 24 7255-7264. (https://doi.org/10.1093/hmg/ddv425)

Kolasa A, Misiakiewicz K, Marchlewicz M \& Wiszniewska B 2012 The generation of spermatogonial stem cells and spermatogonia in mammals. Reproductive Biology 12 5-23. (https://doi.org/10.1016/ S1642-431X(12)60074-6)

Kuramochi-Miyagawa S, Watanabe T, Gotoh K, Takamatsu K, Chuma S, Kojima-Kita K, Shiromoto Y, Asada N, Toyoda A, Fujiyama A et al. 2010 $\mathrm{MVH}$ in piRNA processing and gene silencing of retrotransposons. Genes and Development 24 887-892. (https://doi.org/10.1101/ gad.1902110)

Lien WH, Polak L, Lin M, Lay K, Zheng D \& Fuchs E 2014 In vivo transcriptional governance of hair follicle stem cells by canonical Wnt 
regulators. Nature Cell Biology 16 179-190. (https://doi.org/10.1038/ ncb2903)

Liu CF, Bingham N, Parker K \& Yao HH 2009a Sex-specific roles of betacatenin in mouse gonadal development. Human Molecular Genetics 18 405-417. (https://doi.org/10.1093/hmg/ddn362)

Liu JX, Zhai YH \& Gui JF $2009 b$ Expression pattern of cellular nucleic acidbinding protein (CNBP) during embryogenesis and spermatogenesis of gibel carp. Molecular Biology Reports 36 1491-1496. (https://doi. org/10.1007/s11033-008-9340-2)

Logan CY \& Nusse R 2004 The Wnt signaling pathway in development and disease. Annual Review of Cell and Developmental Biology 20 781-810. (https://doi.org/10.1146/annurev.cellbio.20.010403.113126)

Loh KM, van Amerongen R \& Nusse R 2016 Generating cellular diversity and spatial form: Wnt signaling and the evolution of multicellular animals. Developmental Cell 38 643-655. (https://doi.org/10.1016/j. devcel.2016.08.011)

Margarit E, Armas P, Garcia Siburu N \& Calcaterra NB 2014 CNBP modulates the transcription of Wnt signaling pathway components. Biochimica et Biophysica Acta 1839 1151-1160. (https://doi. org/10.1016/j.bbagrm.2014.08.009)

McLean DJ, Friel PJ, Johnston DS \& Griswold MD 2003 Characterization of spermatogonial stem cell maturation and differentiation in neonatal mice. Biology of Reproduction 69 2085-2091. (https://doi.org/10.1095/ biolreprod.103.017020)

Michelotti EF, Tomonaga T, Krutzsch H \& Levens D 1995 Cellular nucleic acid binding protein regulates the CT element of the human c-myc protooncogene. Journal of Biological Chemistry 270 9494-9499. (https:// doi.org/10.1074/jbc.270.16.9494)

Pfaffl MW 2001 A new mathematical model for relative quantification in real-time RT-PCR. Nucleic Acids Research 29 e45. (https://doi. org/10.1093/nar/29.9.e45)

Sato T, Katagiri K, Gohbara A, Inoue K, Ogonuki N, Ogura A, Kubota Y \& Ogawa T 2011 In vitro production of functional sperm in cultured neonatal mouse testes. Nature 471 504-507. (https://doi.org/10.1038/ nature09850)

Sharpe RM, McKinnell C, Kivlin C \& Fisher JS 2003 Proliferation and functional maturation of Sertoli cells, and their relevance to disorders of testis function in adulthood. Reproduction 125 769-784. (https://doi. org/10.1530/rep.0.1250769)

Shimizu K, Chen W, Ashique AM, Moroi R \& Li YP 2003 Molecular cloning, developmental expression, promoter analysis and functional characterization of the mouse CNBP gene. Gene 307 51-62. (https:// doi.org/10.1016/S0378-1119(03)00406-2)
Svingen T \& Koopman P 2013 Building the mammalian testis: origins, differentiation, and assembly of the component cell populations. Genes and Development 27 2409-2426. (https://doi.org/10.1101/ gad.228080.113)

Wang M, Guo Y, Zhou T, Xue Y, Du G, Wei X, Wang J, Qi L, Zhang H, Li L et al. 2017 The Glial Cell-Derived Neurotrophic Factor (GDNF)responsive phosphoprotein landscape identifies raptor phosphorylation required for spermatogonial progenitor cell proliferation. Molecular and Cellular Proteomics 16 982-997. (https://doi.org/10.1074/mcp. M116.065797)

Yasuda J, Mashiyama S, Makino R, Ohyama S, Sekiya T \& Hayashi K 1995 Cloning and characterization of rat cellular nucleic acid binding protein (CNBP) cDNA. DNA Research 2 45-49. (https://doi.org/10.1093/ dnares/2.1.45)

Zheng B, Zhou Q, Guo Y, Shao B, Zhou T, Wang L, Zhou Z, Sha J, Guo X \& Huang X 2014a Establishment of a proteomic profile associated with gonocyte and spermatogonial stem cell maturation and differentiation in neonatal mice. Proteomics 14 274-285. (https://doi.org/10.1002/ pmic.201300395)

Zheng QS, Wang XN, Wen Q, Zhang Y, Chen SR, Zhang J, Li XX, Sha RN, Hu ZY, Gao F et al. 2014b Wt1 deficiency causes undifferentiated spermatogonia accumulation and meiotic progression disruption in neonatal mice. Reproduction 147 45-52. (https://doi.org/10.1530/REP13-0299)

Zheng B, Zhao D, Zhang P, Shen C, Guo Y, Zhou T, Guo X, Zhou Z \& Sha J 2015 Quantitative proteomics reveals the essential roles of Stromal Interaction Molecule 1 (STIM1) in the testicular cord formation in mouse testis. Molecular and Cellular Proteomics 14 2682-2691. (https://doi. org/10.1074/mcp.M115.049569)

Zhou Q, Guo Y, Zheng B, Shao B, Jiang M, Wang G, Zhou T, Wang L, Zhou Z, Guo X et al. 2015 Establishment of a proteome profile and identification of molecular markers for mouse spermatogonial stem cells. Journal of Cellular and Molecular Medicine 19 521-534. (https:// doi.org/10.1111/jcmm.12407)

Received 30 October 2017

First decision 11 December 2017

Revised manuscript received 1 May 2018

Accepted 8 May 2018 